\title{
Scale-invariant tensor spectrum from conformal gravity
}

\author{
Yun Soo Myund* and Taeyoon Moont \\ Institute of Basic Sciences and Department of Computer Simulation, Inje University \\ Gimhae 621-749, Korea
}

\begin{abstract}
We study cosmological tensor perturbations generated during de Sitter inflation in the conformal gravity with mass parameter $m^{2}=2 H^{2}$. It turns out that tensor power spectrum is scale-invariant.
\end{abstract}

PACS numbers: 98.80.Cq, 04.30.-w, 98.70.Vc

Keywords: inflation, scale-invariant power spectrum, conformal gravity

Typeset Using $\mathrm{AT}_{\mathrm{E} X}$

${ }^{*}$ e-mail address: ysmyung@inje.ac.kr

${ }^{\dagger}$ e-mail address: tymoon@inje.ac.kr 


\section{Introduction}

It is well-known that the Einstein-Weyl gravity has seven propagating gravitational modes, even the massive spin-2 state is a ghost and it violates unitarity [1]. The conformal gravity of $\sqrt{-g} C^{\mu \nu \rho \sigma} C_{\mu \nu \rho \sigma}\left(=C^{2}\right)$ is invariant under the Weyl transformation of $g_{\mu \nu} \rightarrow \Omega^{2}(x) g_{\mu \nu}$ and thus, it has its own interests in gravity and cosmology [2]. The Weyl invariance removes one of seven gravitational modes and it propagates only six modes [3, 4, 5]. In the flat background, these are a healthy massless spin-2, a ghost massless spin-2 and a massless spin-1.

On the other hand, the Einstein-Weyl gravity provides surely massive scalar and vector propagations generated during de Sitter inflation in addition to massive tensor ghost and massless tensor [6, 7, 8, 9]. The authors have shown that in the limit of $m^{2} \rightarrow 0$ (keeping the conformal gravity only), the vector and tensor power spectra became constant [10]. Recently, the Harrison-Zel'dovich scale-invariant spectrum was obtained from the Lee-Wick scalar model in de Sitter spacetime [11. This model is a fourth-order scalar theory whose mass parameter is given by $M^{2}=2 H^{2}$.

It is very important to look for a scale-invariant tensor spectrum which is the most robust and model-independent prediction of inflation. We note that the tensor amplitude is a direct measure of the expansion rate $H$ during inflation. This is in contrast to the scalar amplitude which depends on both $H$ and $\varepsilon$ in the slow-roll inflation. It is worth noting that the Einstein-gravity provides a scale-invariant spectrum in the superhorizon region of $k \ll a H(z \ll 1)$ only. This is known to be a scale-invariant superhorizon spectrum of tensor perturbation.

In this Letter, we will find a scale-invariant tensor power spectrum generated during de Sitter inflation from conformal gravity with mass parameter $m^{2}=2 H^{2}$.

\section{Einstein-Weyl gravity}

We start with the Einstein-Weyl gravity whose action is given by

$$
S_{\mathrm{EW}}=\frac{1}{2 \kappa} \int d^{4} x \sqrt{-g}\left[R-2 \kappa \Lambda-\frac{1}{2 m^{2}} C^{\mu \nu \rho \sigma} C_{\mu \nu \rho \sigma}\right]
$$

where the Weyl-squared term takes the form

$$
C^{\mu \nu \rho \sigma} C_{\mu \nu \rho \sigma}=2\left(R^{\mu \nu} R_{\mu \nu}-\frac{1}{3} R^{2}\right)+\left(R^{\mu \nu \rho \sigma} R_{\mu \nu \rho \sigma}-4 R^{\mu \nu} R_{\mu \nu}+R^{2}\right) .
$$


In the limit of $m^{2} \rightarrow 0$, one recovers the conformal gravity action [12, 10]

$$
S_{\mathrm{CG}}=-\frac{1}{4 \kappa m^{2}} \int d^{4} x \sqrt{-g}\left[C^{\mu \nu \rho \sigma} C_{\mu \nu \rho \sigma}\right]
$$

Here we have $\kappa=8 \pi G=1 / M_{\mathrm{P}}^{2}, M_{\mathrm{P}}$ being the reduced Planck mass and a mass parameter $m^{2}$ is introduced to make the action dimensionless. Greek indices run from 0 to 3 with conventions $(-+++)$, while Latin indices run from 1 to 3 , throughout the paper. Further, we note that the Weyl-squared term $\left(\sqrt{-g} C^{2}\right)$ is invariant under the Weyl transformation of $g_{\mu \nu} \rightarrow \tilde{g}_{\mu \nu}=\Omega^{2}(x) g_{\mu \nu}$. The Einstein equation is given by

$$
G_{\mu \nu}+\kappa \Lambda g_{\mu \nu}-\frac{1}{m^{2}} B_{\mu \nu}=0
$$

where the Einstein tensor $G_{\mu \nu}$ and the Bach tensor $B_{\mu \nu}$ take the forms

$$
\begin{aligned}
G_{\mu \nu} & =R_{\mu \nu}-\frac{1}{2} R g_{\mu \nu} \\
B_{\mu \nu} & =2 \nabla^{\rho} \nabla^{\sigma} C_{\mu \rho \nu \sigma}+G^{\rho \sigma} C_{\mu \rho \nu \sigma} .
\end{aligned}
$$

The solution is given by the de Sitter spacetime whose curvature quantities are

$$
\bar{R}_{\mu \nu \rho \sigma}=H^{2}\left(\bar{g}_{\mu \rho} \bar{g}_{\nu \sigma}-\bar{g}_{\mu \sigma} \bar{g}_{\nu \rho}\right), \quad \bar{R}_{\mu \nu}=3 H^{2} \bar{g}_{\mu \nu}, \quad \bar{R}=12 H^{2}
$$

with $H^{2}=\kappa \Lambda / 3$. We describe de Sitter spacetime explicitly by choosing either a conformal time $\eta$ or a cosmic time $t$

$$
\begin{aligned}
d s_{\mathrm{dS}}^{2}=\bar{g}_{\mu \nu} d x^{\mu} d x^{\nu} & =a(\eta)^{2}\left[-d \eta^{2}+\delta_{i j} d x^{i} d x^{j}\right] \\
& =-d t^{2}+a^{2}(t) \delta_{i j} d x^{i} d x^{j}
\end{aligned}
$$

where the conformal and cosmic scale factors are given by

$$
a(\eta)=-\frac{1}{H \eta}, a(t)=e^{H t}
$$

The choice of Newtonian gauge $B=E=0$ and $\bar{E}_{i}=0$ leads to $10-4=6$ degrees of freedom (DOF)[Considering a fourth-order equation for $h_{i j}$, it amounts 8 DOF]. In this case, the cosmologically perturbed metric can be simplified to be

$$
d s^{2}=a(\eta)^{2}\left[-(1+2 \Psi) d \eta^{2}+2 \Psi_{i} d \eta d x^{i}+\left\{(1+2 \Phi) \delta_{i j}+h_{i j}\right\} d x^{i} d x^{j}\right]
$$


with the transverse vector $\partial_{i} \Psi^{i}=0$ and transverse-traceless tensor $\partial_{i} h^{i j}=h=0$. In order to get the cosmological perturbed equations, one is first to obtain the bilinear action and then, varying it to yield the perturbed equations. We expand the Einstein-Weyl gravity action (11) up to quadratic order in the perturbations of $\Psi, \Phi, \Psi_{i}$, and $h_{i j}$ around the de Sitter background [7. Since the scalar, vector, and tensor are decoupled from each other, we will consider the tensor perturbed equation only

$$
\square^{2} h_{i j}-m^{2} a^{2} \square h_{i j}+2 m^{2} a^{3} H h_{i j}^{\prime}=0,
$$

where $\square=-d^{2} / d \eta^{2}+\partial_{i} \partial^{i}$ and the prime $\left({ }^{\prime}\right)$ denotes the differentiation with respect to $\eta$. We introduce Fourier modes for $h_{i j}$

$$
h_{i j}(\eta, \mathbf{x})=\frac{1}{(2 \pi)^{\frac{3}{2}}} \int d^{3} k \sum_{s=+, \times} p_{i j}^{s}(\mathbf{k}) h_{\mathbf{k}}^{s}(\eta) e^{i \mathbf{k} \cdot \mathbf{x}},
$$

where $p_{i j}^{s}$ is a linear polarization tensor with $p_{i j}^{+/ \times} p^{+/ \times, i j}=1$ and $h_{\mathbf{k}}^{+/ \times}$is a linearly polarized tensor mode. Plugging (13) into (12) leads to the fourth-order differential equation

$$
\left(h_{\mathbf{k}}^{s}\right)^{\prime \prime \prime \prime}+2 k^{2}\left(h_{\mathbf{k}}^{s}\right)^{\prime \prime}+k^{4} h_{\mathbf{k}}^{s}+m^{2} a^{2}\left(h_{\mathbf{k}}^{s}\right)^{\prime \prime}+2 m^{2} a^{3} H\left(h_{\mathbf{k}}^{s}\right)^{\prime}+m^{2} a^{2} k^{2} h_{\mathbf{k}}^{s}=0,
$$

which can be factorized to be

$$
\left[\frac{d^{2}}{d \eta^{2}}-\frac{2}{\eta} \frac{d}{d \eta}+k^{2}\right]\left[\eta^{2} \frac{d^{2}}{d \eta^{2}}-2 \eta \frac{d}{d \eta}+2+k^{2} \eta^{2}+\frac{m^{2}}{H^{2}}\right] h_{\mathbf{k}}^{s}=0
$$

In the limit of $m^{2} \rightarrow \infty$, one recovers the second-order (tensor) equation for the Einstein gravity. In the other limit of $m^{2} \rightarrow 0$, one finds the fourth-order equation for the conformal gravity

$$
\left[\frac{d^{2}}{d \eta^{2}}-\frac{2}{\eta} \frac{d}{d \eta}+k^{2}\right]\left[\eta^{2} \frac{d^{2}}{d \eta^{2}}-2 \eta \frac{d}{d \eta}+2+k^{2} \eta^{2}\right] h_{\mathbf{k}}^{s}=0,
$$

which reduces further to a degenerate fourth-order equation [10]

$$
\left[\frac{d^{2}}{d \eta^{2}}+k^{2}\right]^{2} h_{\mathbf{k}}^{s}=0 \underset{\eta \rightarrow z=-k \eta}{\longrightarrow}\left[\frac{d^{2}}{d z^{2}}+1\right]^{2} h_{\mathbf{k}}^{s}=0
$$

which is equivalent to the equation

$$
\square^{2} h_{i j}=0
$$


for $h_{i j}$. Then, Eq.(16) is considered as the Fourier transform of the Weyl transformation for $\square^{2}$ :

$$
\square^{2} \underset{\eta_{\mu \nu} \rightarrow \bar{g}_{\mu \nu}=a^{2} \eta_{\mu \nu}}{\longrightarrow} \Delta_{4}=\bar{\nabla}^{2}\left(\bar{\nabla}^{2}-2 H^{2}\right), \quad \bar{\nabla}^{2}=\frac{1}{\sqrt{-\bar{g}}} \partial_{\mu}\left(\sqrt{-\bar{g}} \bar{g}^{\mu \nu} \partial_{\mu}\right),
$$

where $\Delta_{4}$ is the fourth-order Lee-Wick operator in the dS background. Also, we note the similar fourth-order equation (59) in Ref.[12] for the covariant approach to the conformal gravity with the transverse gauge. Hence, the fourth-order equation (16) can be interpreted to be the tensor version of the Lee-Wick scalar equation. Accordingly, Eq.(16) implies two second-order equations with $z=-k \eta=k /(a H)$

$$
\begin{array}{r}
{\left[\frac{d^{2}}{d z}-\frac{2}{z} \frac{d}{d z}+1\right] h_{\mathbf{k}}^{s, 1}=0} \\
{\left[\frac{d^{2}}{d z^{2}}-\frac{2}{z} \frac{d}{d z}+1+\frac{2}{z^{2}}\right] h_{\mathbf{k}}^{s, 2}=0}
\end{array}
$$

whose solutions to (20) and (21) are easily found to be

$$
\begin{aligned}
& h_{\mathbf{k}}^{s, 1}=c_{1}(i+z) e^{i z} \\
& h_{\mathbf{k}}^{s, 2}=c_{2} i z e^{i z}
\end{aligned}
$$

where $c_{1}$ and $c_{2}$ are constants to be determined. (22) is the tensor solution to the Einstein gravity [solution to a massless minimally coupled scalar], while (23) is the other solution to the conformal gravity [solution to a massless conformally coupled scalar] in de Sitter spacetime. In this sense, we insist that the conformal gravity includes the Einstein gravity as a part [13].

It is noted that (22) and (23) are also the solutions to the Lee-Wick scalar model [11]. In the Lee-Wick scalar model, its power spectrum is Harrison-Zel'dovich scale-invariant as

$$
\mathcal{P}_{\text {LW }}=\left(\frac{H}{2 \pi}\right)^{2},
$$

whereas the spectrum for a massless minimally coupled scalar takes the form

$$
\mathcal{P}_{\phi}=\left(\frac{H}{2 \pi}\right)^{2}\left[1+\frac{k^{2}}{(a H)^{2}}\right]=\left(\frac{H}{2 \pi}\right)^{2}\left[1+z^{2}\right]
$$

which is obviously a scale-variant spectrum and a scale-invariant superhorizon spectrum.

The tensor power spectrum for the Einstein-Weyl gravity was given by [8]

$$
\mathcal{P}_{\mathrm{EW}}=\frac{2 H^{2}}{\pi^{2} M_{\mathrm{P}}^{2}} \frac{m^{2}}{m^{2}+2 H^{2}}\left(1+z^{2}-\frac{\pi}{2} z^{3}\left|e^{i\left(\frac{\pi \nu}{2}+\frac{\pi}{4}\right)} H_{\nu}^{(1)}(z)\right|^{2}\right), \quad \nu=\sqrt{\frac{1}{4}-\frac{m^{2}}{H^{2}}} .
$$


Here we worry about the negative-norm state because of minus (-) sign in the front of the last term.

Fortunately, in the limit of $m^{2} \rightarrow 0$ and $\kappa\left(=1 / M_{\mathrm{P}}^{2}\right) \rightarrow \infty\left(m^{2} \kappa \rightarrow\right.$ const $)$, one finds the power spectrum being free from negative-norm state as

$$
\mathcal{P}_{\mathrm{EW}}^{m^{2} \rightarrow 0, \kappa \rightarrow \infty}=\frac{m^{2}}{\pi^{2} M_{\mathrm{P}}^{2}}
$$

which implies that the power spectrum of conformal gravity is properly recovered from the Einstein-Weyl gravity when imposing both $m^{2} \rightarrow 0$ and $\kappa \rightarrow \infty$. In the next section, we will obtain (27) by computing the power spectrum of conformal gravity itself.

\section{Conformal gravity}

In order to compute tensor power spectrum in the conformal gravity [10], we begin with the action (3). In this case, there is no restriction on $\mathrm{m}^{2}$. Then, the fourth-order differential equation is given by

$$
\square^{2} h_{i j}=0 \rightarrow\left(h_{\mathbf{k}}^{s}\right)^{\prime \prime \prime \prime}+2 k^{2}\left(h_{\mathbf{k}}^{s}\right)^{\prime \prime}+k^{4} h_{\mathbf{k}}^{s}=0,
$$

which is further rewritten to be (17). This is the same equation for a degenerate PaisUhlenbeck (PU) oscillator [15, 16] and its solution is given by

$$
h_{\mathbf{k}}^{s}(z)=\frac{N}{2 k^{2}}\left[i\left(a_{2}^{s}+a_{1}^{s} z\right) e^{i z}+c . c .\right]
$$

with $N$ the normalization constant. After quantization, $a_{2}^{s}$ and $a_{1}^{s}$ are promoted to operators $\hat{a}_{2}^{s}(\mathbf{k})$ and $\hat{a}_{1}^{s}(\mathbf{k})$, which leads to the expression $\hat{h}_{\mathbf{k}}^{s}(z)$. The presence of $z$ in $(\cdots)$ reflects clearly that $h_{\mathbf{k}}^{s}(z)$ is a solution to the degenerate equation (17). Together with $N=\sqrt{2 \kappa m^{2}}$, the canonical quantization could be accomplished by introducing commutation relations between $\hat{a}_{i}^{s}(\mathbf{k})$ and $\hat{a}_{j}^{s \dagger}\left(\mathbf{k}^{\prime}\right)$ as

$$
\left[\hat{a}_{i}^{s}(\mathbf{k}), \hat{a}_{j}^{s^{\prime} \dagger}\left(\mathbf{k}^{\prime}\right)\right]=2 k \delta^{s s^{\prime}}\left(\begin{array}{cc}
0 & -i \\
i & 1
\end{array}\right) \delta^{3}\left(\mathbf{k}-\mathbf{k}^{\prime}\right) .
$$

The tensor power spectrum is defined by

$$
\left\langle 0\left|\hat{h}_{i j}(\eta, \mathbf{x}) \hat{h}^{i j}\left(\eta, \mathbf{x}^{\prime}\right)\right| 0\right\rangle=\int d^{3} k \frac{\mathcal{P}_{\mathrm{h}}^{m^{2}}}{4 \pi k^{3}} e^{i \mathbf{k} \cdot\left(\mathbf{x}-\mathbf{x}^{\prime}\right)} .
$$


Here we choose the Bunch-Davies vacuum $|0\rangle$ by imposing $\hat{a}_{i}^{s}(\mathbf{k})|0\rangle=0$. Using the definition

$$
\mathcal{P}_{\mathrm{h}}^{m^{2}} e^{i \mathbf{k} \cdot\left(\mathbf{x}-\mathbf{x}^{\prime}\right)} \equiv \sum_{s, s^{\prime}=+, \times} \mathcal{P}_{\mathrm{h}}^{s s^{\prime}, m^{2}}
$$

and substituting (13) together with $\hat{h}_{\mathbf{k}}^{s}(z)$ into (31), then one finds that $\mathcal{P}_{\mathrm{h}}^{s s^{\prime}, m^{2}}$ takes the form

$$
\begin{aligned}
\mathcal{P}_{\mathrm{h}}^{s s^{\prime}, m^{2}}= & \frac{m^{2} k}{4 \pi^{2} M_{\mathrm{P}}^{2}} \int d^{3} \mathbf{k}^{\prime}\left[\frac{1}{k^{2}} p_{i j}^{s}(\mathbf{k}) p^{i j s^{\prime}}\left(\mathbf{k}^{\prime}\right) \times\langle 0|\left(\left[\hat{a}_{2}^{s}(\mathbf{k}), \hat{a}_{2}^{s^{\prime} \dagger}\left(\mathbf{k}^{\prime}\right)\right]+z\left[\hat{a}_{2}^{s}(\mathbf{k}), \hat{a}_{1}^{s^{\prime} \dagger}\left(\mathbf{k}^{\prime}\right)\right]\right.\right. \\
& \left.\left.\quad+z\left[\hat{a}_{1}^{s}(\mathbf{k}), \hat{a}_{2}^{s^{\prime} \dagger}\left(\mathbf{k}^{\prime}\right)\right]+z^{2}\left[\hat{a}_{1}^{s}(\mathbf{k}), \hat{a}_{1}^{s^{\prime} \dagger}\left(\mathbf{k}^{\prime}\right)\right]\right)|0\rangle e^{i\left(\mathbf{k} \cdot \mathbf{x}-\mathbf{k}^{\prime} \cdot \mathbf{x}^{\prime}\right)}\right] \\
= & \frac{m^{2} k}{4 \pi^{2} M_{\mathrm{P}}^{2}} \int d^{3} \mathbf{k}^{\prime}\left[\frac{1}{k^{\prime 2}} p_{i j}^{s}(\mathbf{k}) p^{i j s^{\prime}}\left(\mathbf{k}^{\prime}\right) \times\left\langle 0\left|\left[\hat{a}_{2}^{s}(\mathbf{k}), \hat{a}_{2}^{s^{\prime} \dagger}\left(\mathbf{k}^{\prime}\right)\right]\right| 0\right\rangle e^{i\left(\mathbf{k} \cdot \mathbf{x}-\mathbf{k}^{\prime} \cdot \mathbf{x}^{\prime}\right)}\right] \\
= & \frac{m^{2}}{2 \pi^{2} M_{\mathrm{P}}^{2}} p_{i j}^{s} p^{i j s^{\prime}} \delta^{s s^{\prime}} e^{i \mathbf{k} \cdot\left(\mathbf{x}-\mathbf{x}^{\prime}\right)} .
\end{aligned}
$$

In obtaining (35), we used the commutation relations of (30) which reflect the quantum nature of a degenerate PU oscillator. This is in contrast to a non-degenerate PU oscillator for the Lee-Wick scalar theory [11].

Finally, from (32) and (35), we obtain the tensor power spectrum

$$
\mathcal{P}_{\mathrm{h}}^{m^{2}}=\frac{m^{2}}{\pi^{2} M_{\mathrm{P}}^{2}},
$$

which is the same form as in (27).

Further, by choosing $m^{2}=2 H^{2}$ specifically, (36) reduces to

$$
\mathcal{P}_{\mathrm{h}}^{m^{2}=2 H^{2}}=\frac{2 H^{2}}{\pi^{2} M_{\mathrm{P}}^{2}}
$$

which is the scale-invariant tensor spectrum. This is surely compared to the scale-variant tensor spectrum for the Einstein gravity [17]

$$
P_{\mathrm{h}}(k)=\frac{2 H^{2}}{\pi^{2} M_{\mathrm{P}}^{2}}\left(1+\frac{k^{2}}{(a H)^{2}}\right)
$$

during de Sitter inflation. It is worth noting that one could not find the scale-invariant form of (37) from the general expression (36) unless one chooses $m^{2}=2 H^{2}$. This implies that there is an ambiguity in determining a correct amplitude of the power spectrum for conformal gravity. 


\section{Discussions}

We have found the scale-invariant tensor power spectrum generated during de Sitter inflation from conformal gravity with mass parameter $m^{2}=2 H^{2}$. This scale-invariant tensor spectrum could be understood because the conformal gravity is invariant under the Weyl transformation. This contrasts to the scale-variant tensor spectrum (38) of Einstein gravity which is not Weyl-invariant. This is considered as a tensor version of Harrison-Zel'dovich scale-invariant spectrum obtained from the Lee-Wick scalar model with mass parameter $M^{2}=2 H^{2}$. We summarize below the difference between second-order theory and fourthorder theory and similarity between scalar and tensor in the same order. Their DOF are shown explicitly.

\begin{tabular}{|c|c|c|}
\hline power spectrum & scalar theory (DOF) & tensor theory (DOF) \\
\hline scale-variant & massless minimally coupled & Einstein gravity (2) \\
(second-order) & scalar theory (1) & \\
\hline scale-invariant & Lee-Wick model & Conformal gravity \\
(fourth-order) & with $M^{2}=2 H^{2}(2)$ & with $m^{2}=2 H^{2}(4)$ \\
\hline
\end{tabular}

Finally, we would like to explain why our computation is meaningful even in the presence of ghost. As was shown in (28), the conformal gravity provides a healthy massless spin-2 and an unhealthy (ghost) massless spin-2. In computing the power spectrum, one has to distinguish higher-time derivative terms (ghost) from ghost state (negative-norm state). In the case of the Lee-Wick model, its scalar power spectrum based on the quantization scheme of non-degenerate PU oscillator is given by $\mathcal{P}_{\mathrm{LW}}=(H / 2 \pi)^{2}\left[1+(k \eta)^{2}-(k \eta)^{2}\right]$ where the first two are contribution from a massless minimally scalar and the last from a massless conformally coupled scalar when fourth-order LW operator is partially factorized into two second-order operators with minus sign in front of the massless conformally coupled scalar operator. Here there is no negative-norm (ghost state) for $\mathcal{P}_{\text {LW }}$ because the second term cancels against the third term. This explains why the LW computation is meaningful even in the presence of ghost. Similarly, the power spectrum of conformal gravity based on the quantization scheme (30) of degenerate PU oscillator (29) is given by (36) which is free from negative-norm state (ghost state) and scale-invariant. This implies that offdiagonal cancellation of $z$-order in (33) using (30) is important to have positive-norm state. 
Consequently, our whole computation is safe even in the presence of ghost because the power spectrum of conformal gravity (36) [equivalently, (27)] is free from ghost state.

\section{Acknowledgement}

This work was supported by the National Research Foundation of Korea (NRF) grant funded by the Korea government (MEST) (No.2012-R1A1A2A10040499). 


\section{References}

[1] K. S. Stelle, Phys. Rev. D 16, 953 (1977).

[2] R. J. Riegert, Phys. Rev. Lett. 53, 315 (1984).

[3] S. Deser, E. Joung and A. Waldron, J. Phys. A 46, 214019 (2013) arXiv:1208.1307 [hep-th]].

[4] S. Deser, E. Joung and A. Waldron, Phys. Rev. D 86, 104004 (2012) arXiv:1301.4181 [hep-th]].

[5] S. F. Hassan, A. Schmidt-May and M. von Strauss, arXiv:1303.6940 [hep-th].

[6] T. Clunan and M. Sasaki, Class. Quant. Grav. 27, 165014 (2010) arXiv:0907.3868 [hep-th]].

[7] N. Deruelle, M. Sasaki, Y. Sendouda and A. Youssef, JCAP 1103, 040 (2011) arXiv:1012.5202 [gr-qc]].

[8] N. Deruelle, M. Sasaki, Y. Sendouda and A. Youssef, JHEP 1209, 009 (2012) arXiv:1202.3131 [gr-qc]].

[9] Y. S. Myung and T. Moon, JCAP 1408, 061 (2014) [arXiv:1406.4367 [gr-qc]].

[10] Y. S. Myung and T. Moon, arXiv:1407.0441 [gr-qc].

[11] Y. S. Myung and T. Moon, arXiv:1412.7263 [gr-qc].

[12] P. D. Mannheim, Phys. Rev. D 85, 124008 (2012) [arXiv:1109.4119 [gr-qc]].

[13] J. Maldacena, arXiv:1105.5632 [hep-th].

[14] K. Dimopoulos, Phys. Rev. D 74, 083502 (2006) hep-ph/0607229].

[15] P. D. Mannheim and A. Davidson, Phys. Rev. A 71, 042110 (2005) hep-th/0408104.

[16] Y. W. Kim, Y. S. Myung and Y. J. Park, Phys. Rev. D 88, 085032 (2013) arXiv:1307.6932.

[17] D. Baumann, arXiv:0907.5424 [hep-th]. 